\title{
A Highly Efficient Polarized Superfluorescent Fiber Source for Fiber-Optic Gyroscope Applications
}

\author{
H. C. Su and Lon A. Wang
}

\begin{abstract}
We demonstrate a novel polarized superfluorescent fiber source (SFS) that increases the output power in the desired polarization by a factor of 1.96 over that of an unpolarized SFS at the same pump. This is achieved by inserting a polarizer at the optimal point in the bidirectionally pumped Erbium-doped fiber of an unpolarized SFS. Stable pump-power-dependent mean wavelength operation and less sensitivity to the insertion loss, important for fiber-optic gyroscope applications, are obtained in this configuration.
\end{abstract}

Index Terms-Fiber-optic gyroscope (FOG), polarized fiber source, superfluorescent fiber source (SFS).

\section{INTRODUCTION}

$\mathbf{E}$ RBIUM-DOPED superfluorescent fiber sources (SFSs) have become popular in fiber-optic gyroscope (FOG) applications because of their high output power and broad-band spectral characteristics [1]. In a high-grade FOG, light from a source must pass through a polarizer before entering the sensing coil. When an unpolarized SFS is used, half of its power is lost at the input of the sensing coil. If an SFS is capable of generating a linearly polarized output instead, this loss can be eliminated.

To evaluate the polarization conversion efficiency, a figure of merit $k$ is defined as the ratio of the output power in the desired polarization for an SFS using a polarizer to that in the same polarization without a polarizer. Polarized SFSs in the single-pass backward (SPB) configuration have been demonstrated to have a high $k$ value of 1.76 [2]. This performance requires a polarizing-fiber loss under $0.55 \mathrm{~dB}$ [2]. However, the degradation of its performance is very sensitive to the polarizing-fiber's insertion loss. A 3-dB polarizer loss will lower the $k$ value to unity, which has no improvement at all. We have found that polarized double-pass backward (DPB) SFSs are not only highly effective in polarization conversion $(k=1.88)$ but also less sensitive to the insertion loss of the polarizer [3]. The experiment setup of the polarized DPB SFS is shown in Fig. 1(a). The polarizer is placed as close to the mirror end as possible, resulting in its less sensitivity to the insertion loss. The polarized backward amplified spontaneous emission (ASE), after passing the polarizer, consumes most gain and becomes the dominant component of the output power for the polarized DPB SFS. However, some weak unpolarized backward ASE still survives at the presence

Manuscript received November 4, 2002; revised May 16, 2003. This work was supported in part by the National Science Council Taiwan, R.O.C., under Contract NSC 91-2215-E-002-028 and in part by the Education Ministry, Taiwan, R.O.C., under Contract 89-E-FA06-2-4-7.

The authors are with the Institute of Electro-Optical Engineering and the Department of Electrical Engineering, National Taiwan University, Taipei 106,

Taiwan, R.O.C. (e-mail: lon@ccms.ntu.edu.tw).

Digital Object Identifier 10.1109/LPT.2003.816126

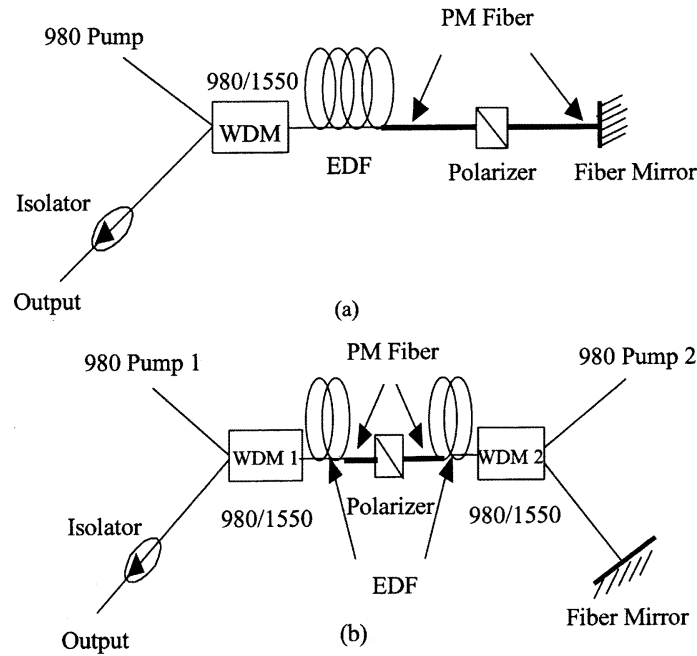

Fig. 1. Schematic diagram of polarized (a) DPB and (b) BPDP SFSs

of strong gain competition. This weak unpolarized ASE noise degrades the output extinction ratio (ER), which is defined as the ratio of the power in the desired polarization to the orthogonal component. The $k$ value is, hence, limited due to these unpolarized backward ASE signals. One possible way to enhance the output ER of the polarized DPB SFS is to launch part of the total pump power at the fiber mirror end. Additional backward ASE, thus generated by the second pump source, becomes polarized after the polarizer; therefore, the output ER is enhanced due to more severe suppression of unpolarized ASE in the highly inverted region. Besides, the ASE distribution of a bidirectionally pumped EDF is different from that of a single-pumped EDF. The optimal polarizer position for the bidirectionally pumped double-pass (BPDP) SFS to have the least sensitivity to insertion loss will be at somewhere in the middle of the EDF length, where the attenuation of both ASE and pump effected by the polarizer is the least. Since the EDF length between the polarizer and the output end is shortened, the output ER will, thus, be further enhanced due to the reduction in unpolarized backward ASE.

Since the stability of the mean wavelength determines the accuracy of rotation detection, it is regarded as one of the most important parameters for a high-grade FOG. The variation of mean wavelength with temperature is affected by three contributing sources: intrinsic thermal effect, pump-wavelength, and pumppower related factors [4]. Since the first and the second factors are related to the materials and pump diodes, not within our capability, we will focus on minimizing the variation of the pump-power-dependent mean wavelength of SFSs. The polarized DPB SFS has been reported capable of operating with a 
TABLE I

EDF LENGTHS AND CORRESPONDING UNPOLARIZED OUTPUT POWER FOR THE BPDP SFS PUMPED AT VARIOUS PUMP-POWER RATIOS FOR A TOTAL 100-mW POWER

\begin{tabular}{c|c|c|c|c}
\hline $\begin{array}{c}\text { Pump Ratio, } \\
\text { P1/(P1+P2) }\end{array}$ & 0.7 & 0.8 & 0.9 & 1.0 \\
\hline EDF Length (m) & 24 & 22 & 18 & 13 \\
\hline $\begin{array}{c}\text { Unpolarized } \\
\text { Output Power } \\
(\mathrm{mW})\end{array}$ & 20.74 & 23.62 & 26.63 & 27.52 \\
\hline
\end{tabular}

stable pump-power-dependent mean wavelength [3]. Any adjustment in the polarized DPB SFS for enhancing the $k$ value, however, must also conserve this characteristic.

\section{Simulation AND EXPERIMENT RESUlts}

Our novel configuration is shown in Fig. 1(b). Pump 2 means that some fraction of total pump power is launched at the fiber mirror end, not necessarily to use a second pump diode. To optimize its performance, EDF length, the pump ratio, which is defined to be Pump 1 over total pump power, and polarizer position are three parameters to be found. Simulation is used to illustrate the design process and to explain some results physically. Since we do not have the cross sections of the EDF used in our experiment, the parameters given in [4] are utilized in simulation. Simulation results may have some differences from the experimental ones; however, they are shown to agree well in trend.

The $k$ value of the polarized DPB SFS is enhanced by utilizing the bidirectional-pumping configuration. However, adding the second pump to the DPB SFS may destroy its pump-power-independent mean wavelength operation. This is because the backward ASE generated by the second pump source only experiences a single pass in EDF to reach the output end. Compared with the DPB SFS at the same total pump power, the effective path length of total ASE traveling in EDF decreases for the BPDP SFS. As the total pump power goes higher, the optimized EDF length for the DPB SFS is not enough for the BPDP SFS to absorb the increasing ASE near $1530 \mathrm{~nm}$. Therefore, the mean wavelength will decrease with the increasing total pump power. To recover this characteristic, a longer EDF length is needed to absorb the ASE at the wavelength near $1530 \mathrm{~nm}$. The more the pump power at the fiber mirror end, the more the EDF length is needed. The mean wavelength stability is considered around $100-\mathrm{mW}$ total pump power, which is comparable with our experiment parameters. Mean wavelength stability is evaluated in parts per million per watt, which is defined in [4]. Simulation results of the effect of pump ratio on optimized EDF length to achieve mean wavelength variation less than $1 \mathrm{ppm} / \mathrm{mW}$ around $100-\mathrm{mW}$ total pump power are listed in Table I. Optimized EDF lengths and corresponding unpolarized output power of the BPDP SFS pumped at different ratios for 100-mW total pump power are also shown in Table I. In simulation, the wavelength-division-multiplexing (WDM) couplers and the isolator are assumed to be lossless and $90 \%$ reflectance for the fiber mirror. It is noted that the output power is lower for the BPDP SFS with lower pump ratio because of more ASE absorption in a longer EDF.



Fig. 2. Simulated $k$ values for various polarizer insertion losses at different polarizer positions for the BPDP SFS.

For the BPDP SFS with pump ratio lower than 0.9 at the same total pump, its unpolarized output power is lower than the output power in the dominant polarization of the polarized DPB SFS. Even with perfect polarization conversion, polarized BPDP SFSs with pump ratio lower than 0.9 cannot generate as high polarized output power as the polarized DPB SFS can. Therefore, we choose the BPDP SFS with 18-m EDF and pump ratio of 0.9 to further optimize its polarizer position. Simulated $k$ values for various insertion losses at different polarizer positions are shown in Fig. 2. The initial polarizer position is placed at the first WDM and the ending one at the second WDM. It is noted that placing the polarizer near the EDF ends will cause degradation of the $k$ value because relatively high pump and ASE power are attenuated by the polarizer. The optimal position of the polarizer for the highest $k$ and for being least sensitive to insertion loss for the polarized BPDP SFS is $\sim 11.5 \mathrm{~m}$ away from the first WDM, where both pump and ASE power are lower than they are at EDF ends. The output power of the polarized BPDP SFS in dominant polarization is $26.1 \mathrm{~mW}$ with corresponding $k$ value of 1.96 when a polarizer is placed at this optimal position.

Some experiments are made to confirm the simulation results. Since we have no tunable coupler to tune the pump ratio of the pump power launched at two EDF ends, two 980 -nm laser diodes are used in our experiments. Two 980/1550-nm WDM couplers are used for combining and coupling pump and ASE signals. The single-mode EDF (DF 1500, FIBERCORE) has a cutoff wavelength of $945 \mathrm{~nm}$ and a numerical aperture of 0.23 . The absorption of EDF we used is 9 and $13.5 \mathrm{~dB} / \mathrm{m}$ at 979 and $1531 \mathrm{~nm}$, respectively. The ER of the in-line polarizer (Wave Optics 650/967-0700) is about $30 \mathrm{~dB}$ with an insertion loss of $\sim 0.4 \mathrm{~dB}$. A fiber mirror $(R \sim 85 \%)$ is utilized to reverse the forward ASE signals to the backward direction. The output spectra and optical power of the backward ASE signals are measured by an optical spectrum analyzer. A polarization analyzer is used to measure the output ER.

Since the parameters of EDF used in the simulation and experiments are not the same, the optimal EDF length for the pump-power-independent mean wavelength operation and the optimal polarizer position for least sensitivity to insertion loss had to be adjusted in the experiments. The EDF length of DPB SFS is chosen to be $11 \mathrm{~m}$ while a $15-\mathrm{m}$ EDF is used in the BPDP 




Fig. 3. Measured pump-power-dependent output ER and $k$ value for the polarized DPB and BPDP SFSs.

SFS with pump ratio of 0.9 to achieve the pump-power-independent mean wavelength operation around $120-\mathrm{mW}$ total pump power. For the polarized BPDP SFS, the polarizer is inserted at the position $9.5 \mathrm{~m}$ away from the first WDM to obtain the highest polarization conversion efficiency and least sensitivity to insertion loss. The following measured results are based on the parameters described above.

The unpolarized output power of the BPDP SFS with $15-\mathrm{m}$ EDF at pump ratio of 0.9 for $120-\mathrm{mW}$ total pump power is $24.52 \mathrm{~mW}$. With a polarizer placed at the optimal position, $24.2-\mathrm{mW}$ polarized output power with an ER of $22.4 \mathrm{~dB}$ is measured at the same pump. No obvious fluctuations in state of polarization are observed if the coils of EDF and the components used in the experiments are kept still. Its output power in the dominant polarization is $24.06 \mathrm{~mW}$. The $k$ value is, thus, calculated to be 1.96. Similar experiments are made for the DPB SFS. The unpolarized and polarized output power for the DPB SFS at $120-\mathrm{mW}$ total pump power are 24.32 and $23.86 \mathrm{~mW}$, respectively. Its output ER is $13.3 \mathrm{~dB}$ and the corresponding $k$ value is 1.88 . Measured output ER and the $k$ value for the polarized BPDP and DPB SFSs at various pump power are shown in Fig. 3. The output ER (or the $k$ value) of the polarized BPDP SFS is higher than that of the polarized DPB SFS at the same pump power because of the reason described in introduction. At high pump power, the $k$ values for both configurations seem to be at saturated maximum levels. It is noted that a smaller pump is needed to reach the maximum of $k$ for the polarized BPDP SFS. This is because polarized backward ASE induced by the second pump source can enhance the suppression of unpolarized ASE in highly inverted EDF region near the first WDM. Therefore, a lower pump is required for the polarized BPDP SFS to suppress unpolarized ASE to noise level, which leads to the saturated maximum $k$ value.

Both measured pump-power-dependent mean wavelength and linewidth for the polarized BPDP and DPB SFSs are shown in Fig. 4. Both configurations have been experimentally demonstrated to have stable pump-power-independent mean wavelength characteristic around $120-\mathrm{mW}$ pump power. The

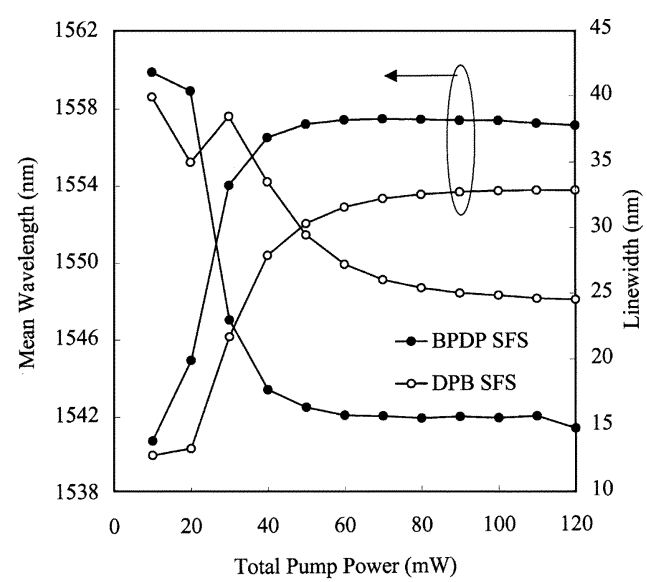

Fig. 4. Measured pump-power-dependent mean wavelength and linewidth for the polarized DPB and BPDP SFSs.

mean wavelength is larger for the polarized BPDP SFS than that of the polarized DPB SFS because of the use of longer EDF, which absorbs more ASE signals near the wavelength near $1530 \mathrm{~nm}$. The definition of linewidth for SFS is given in [5]. The linewidth decreases as the pump power increases because the 1550-nm peak grows faster than the 1530-nm peak. The linewidth of the polarized BPDP SFS at $120-\mathrm{mW}$ pump power is $14.77 \mathrm{~nm}$. Although it is smaller than that of the polarized DPB SFS, it is still enough for applications of navigation-grade FOG [4].

Insertion loss of the polarizer will degrade the $k$ value of the polarized SFS [3]; therefore, such degradation is also studied as a tunable optical attenuator is inserted between the polarizer and EDF to tune the insertion loss. The $k$ values for the polarized BPDP SFS without the optical attenuator at 120-mW pump power is 1.96. With an insertion loss as large as $5 \mathrm{~dB}$, the polarized BPDP SFS still possesses $k$ value of 1.74 . The approximate insertion-loss dependent $k$ value degradation slope for the polarized BPDP SFS is $-0.044 / \mathrm{dB}$. Note the backward ASE generated by the second pump source only passed the polarizer once, resulting in less ASE power being attenuated by the polarizer, and thus, is less sensitive to polarizer insertion loss.

\section{REFERENCES}

[1] N. Duling, R. P. Moeller, W. K. Burns, C. A. Villarruel, L. Goldberg, E. Snitzer, and H. Po, "Output characteristics of diode pumped fiber ASE sources," IEEE J. Quantum Electron., vol. 27, pp. 995-1003, Apr. 1991.

[2] D. G. Falquier, J. L. Wagener, M. J. F. Digonnet, and H. J. Shaw, "Polarized superfluorescent fiber source," Opt. Lett., vol. 22, pp. 160-162, 1997.

[3] L. A. Wang, C. D. Lee, H. C. Su, and J. F. Chang, "An insertion loss insensitive and mean wavelength stable polarized superfluorescent fiber source," in Proc. OFC, 2002, Paper ThGG 16, pp. 633-634.

[4] P. F. Wysocki, M. J. F. Digonet, B. Y. Kim, and H. J. Shaw, "Characteristics of erbium-doped superfluorescent fiber sources for interferometric sensor application,” J. Lightwave Technol., vol. 12, pp. 550-567, Mar. 1994.

[5] W. K. Burns, R. P. Moeller, and A. Dandridge, "Excess noise in fiber gyroscope sources," IEEE Phonton. Technol. Lett., vol. 2, pp. 606-608, Aug. 1990. 